\title{
The harder one looks, the more one finds
}

\author{
Michael J. Mack, MD
}

\author{
From the Department of Cardiac Surgery, Heart Hospital Baylor Plano, Baylor Scott \& White Health, Dallas, Tex. \\ Disclosures: Author has nothing to disclose with regard to commercial support. \\ Received for publication March 7, 2016; accepted for publication March 10, 2016; available ahead of print April \\ $18,2016$. \\ Address for reprints: Michael J. Mack, MD, Department of Cardiac Surgery, Heart Hospital Baylor Plano, Baylor \\ Scott \& White Health, 1100 Allied Dr, Plano, TX 75075 (E-mail: michael.mack@ baylorhealth.edu). \\ J Thorac Cardiovasc Surg 2016;152:5-6 \\ 0022-5223/ $\$ 36.00$ \\ Copyright (c) 2016 by The American Association for Thoracic Surgery \\ http://dx.doi.org/10.1016/j.jtcvs.2016.03.041
}

The article by Gleason and colleagues ${ }^{1}$ in this issue of the Journal reports the risk and extent of neurologic injury in high risk patients with aortic stenosis undergoing either transcatheter aortic valve replacement (TAVR) or surgical aortic valve replacement (SAVR). The study reports the rate of stroke at 30 days, 1 year, and 2 years postprocedure in the 750 patients enrolled in the Corevalve High-Risk Study. They found no difference in stroke between the TAVR and SAVR groups at 30 days and 1 year, but a higher incidence of stroke at 2 years in the group undergoing SAVR.

The authors also performed a prespecified neurologic substudy in a selected group of patients, 199 of 750 $(27 \%)$, involving various assessments of neurologic and cognitive function, including the National Institutes of Health Stroke Scale, the Mini-Mental State Exam, new gait abnormality, weakness in left and right hand motor function, and writing and drawing evaluations. Unfortunately, it is difficult to assess the information gleaned from this substudy. The authors provide no information on how the patients were selected, and there was a disproportionate number of TAVR versus SAVR patients enrolled (111 vs 88). Although all patients were tested at baseline, the proportion tested at discharge ranged between $45 \%$ and $74 \%$ and that tested at 1 year ranged between $36 \%$ and $53 \%$ for the 7 tests. Compounding this missingness of data is the possibility of survivorship bias and the knowledge that patients with neurologic dysfunction are those least likely to return for follow-up.

What is the magnitude of neurologic injury after both TAVR and SAVR? Well, it depends on the definition of stroke, who is diagnosing it, when are they looking, and how they are diagnosing it. The American Heart Association/American Stroke Association consensus definition of stroke includes "imaging evidence of a CNS infarction with or without acute neurological dysfunction." ${ }^{2}$ We also know that cerebral embolization occurs in virtually all patients undergoing both SAVR and TAVR. Diffusion-weighted magnetic resonance imaging (DW MRI) has identified evidence of neurologic infarction in

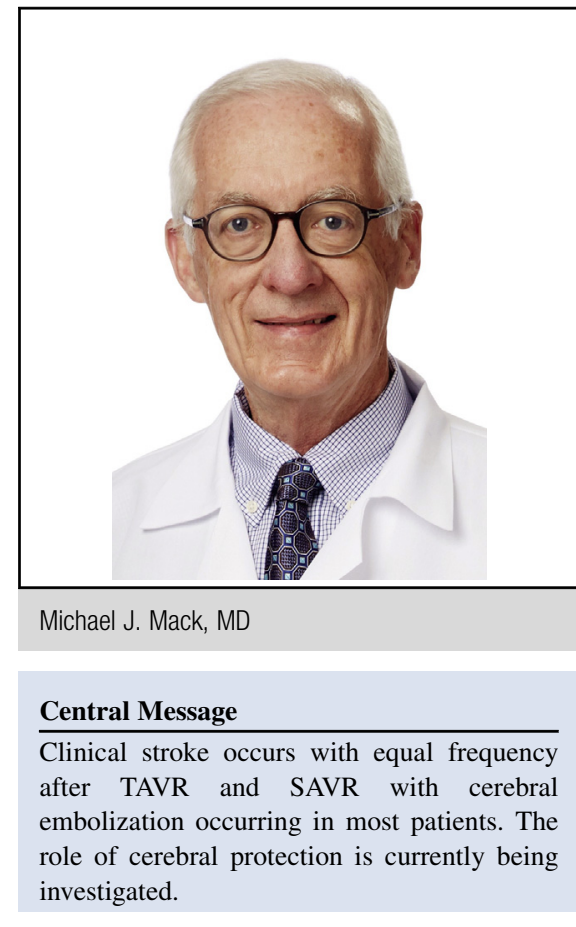

See Article page 85 .

See Editorial Commentary page 97.

$58 \%$ to $100 \%$ of patients undergoing TAVR (Figure 1 ). Furthermore, silent infarcts in other settings are known to be associated with several adverse neurologic and cognitive consequences, including impaired mobility, physical decline, depression, cognitive dysfunction, dementia, and Alzheimer's disease. ${ }^{4}$

We also know that the harder one looks and the closer the patient is examined postprocedure, the more neurologic abnormalities are discovered. As demonstrated by Messe and colleagues, ${ }^{5}$ examination of postsurgical patients by a neurologist doubled the rate of detection of clinically evident stroke compared with examination by other clinicians. Despite some early concerns about a higher incidence of stroke after TAVR, it appears that rates are relatively equal between TAVR and SAVR, as demonstrated in this study. The higher early stroke rate in TAVR compared with SAVR in the early trials has consistently diminished, and the rate now appears to be equal to that of SAVR with or without neurologist evaluation. It is also not clear what assessment tools should be used. We know, for example, that the National Institutes of Health Stroke Scale assesses $<10 \%$ of the cognitive function of the brain. 


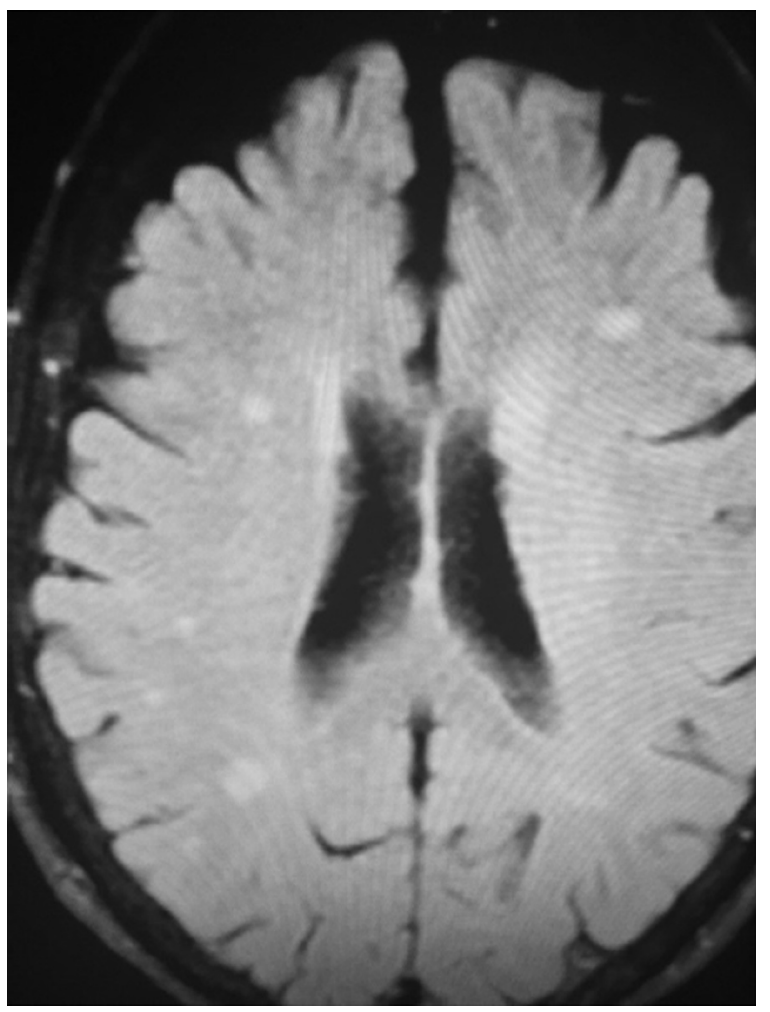

FIGURE 1. Typical DW MRI findings of multiple emboli in a patient without neurologic deficit following a TAVR procedure.

What is not clear is the role that cerebral protection will serve after TAVR and SAVR. Randomized trials after both procedures have demonstrated that devices that capture, deflect, or aspirate emboli are capable of decreasing both the number and volume of DW MRI-detected lesions in the brain. A number of ongoing studies of TAVR, including the SENTINEL (ClinicalTrials.gov identifier NCT02214277) (emboli capture) and REFLECT (NCT02536196) (emboli deflection) studies, may help answer these questions. Likewise, an ongoing study of 530 patients undergoing SAVR in the Cardiothoracic Surgery Trials Network jointly sponsored by the National Heart Lung and Blood Institute and National Institute of Neurologic Disorders and Stroke Neuroprotection study (NCT02389894) has completed approximately $40 \%$ of planned enrollment. All of these trials include data on clinical stroke evaluation, neurocognitive assessment, and DW MRI for all patients.

What is also evident from the Gleason study is that the majority of neurologic events occurred outside of the immediate 30-day postprocedure period. There are a number of possible explanations for this finding. First, this cohort is clearly a risk-prone population. Second, the incidence of stroke after the first 30 days was higher after
SAVR than after TAVR. The percentage of patients discharged on dual antiplatelet therapy (DAPT) or anticoagulation was higher in the patients undergoing TAVR compared with those undergoing SAVR. This could be indicative of a possible protective effect against stroke. We also know that the incidence of postoperative atrial fibrillation is higher after SAVR than after TAVR, perhaps also contributing to the long-term increased risk of stroke after SAVR and suggesting a possible role for wider use of DAPT or anticoagulation. A number of trials of DAPT and novel anticoagulants after TAVR are commencing as well.

In an effort to add structure and order to the field, the Academic Research Consortium (ARC) has convened a multidisciplinary consortium to propose common definitions, assessment tools, and trial endpoints. Led by Alexandra Lansky, the effort, termed Neuro ARC, includes neurologists, neuropsychologists, cardiologists, cardiac surgeons, neuroimaging specialists, device manufacturers, and the Food and Drug Administration.

In summary, the incidence of frank, clinical stroke after TAVR is decreasing and is likely equal to that of SAVR. However, the harder one looks, the more one finds depending on who assesses the patient, when that assessment is done, and what tools are used. It is also clear that virtually all patients have evidence of cerebral embolization after TAVR and SAVR. There is evidence that embolic protection devices reduce cerebral embolization, that cerebral embolization is associated with early neurocognitive abnormalities, and that cerebral embolization is associated with long-term cognitive decline and dementia. The embolic protection and antiplatelet/anticoagulation trials in patients undergoing TAVR and SAVR now underway will help identify whether reducing embolization and postprocedure therapy are associated with improved clinical neurologic outcomes.

\section{References}

1. Gleason TG, Schindler JT, Adams DH, Reardon MJ, Kleiman NS, Caplan LR, et al. The risk and extent of neurological events are 1 equivalent for high-risk patients treated with transcatheter or surgical aortic valve replacement. $J$ Thorac Cardiovasc Surg. 2016;152:85-96.

2. Sacco RL, Kasner SE, Broderick JP, Caplan LR, Connors JJ, Culebras A, et al. An updated definition of stroke for the 21st century: a statement for healthcare professionals from the American Heart Association/American Stroke Association. Stroke. 2013;44:2064-89.

3. Kahlert P, Knipp SC, Schlamann M, Thielman M, Al-Rashid F, Weber M, et al. Silent and apparent cerebral ischemia after percutaneous transfemoral aortic valve implantation: a diffusion-weighted magnetic resonance imaging study. Circulation. 2010;121:870-8.

4. Arvanitakis Z, Leurgans SE, Barnes LL, Bennett DA, Schneider JA. Microinfarct pathology, dementia, and cognitive systems. Stroke. 2011;42:722-7.

5. Messe SR, Acker MA, Kasner SE, Fanning M, Giovannetti T, Ratcliffe SJ, et al. Stroke after aortic valve surgery: results from a prospective cohort. Circulation. 2014;129:2253-61. 